\title{
On Information AND COMPETITION in Private Value Auctions
}

\author{
Juan-José Ganuza* and José S. Penalva ${ }^{\dagger}$ \\ January 17, 2006
}

\begin{abstract}
This paper studies the relationship between the auctioneer's provision of information and the level of competition in private value auctions. We use a general notion of informativeness which allows us to compare the efficient with the (privately) optimal amount of information provided by the auctioneer. We show that in the private value setting more information increases the efficiency of the allocation while it also increases informational rents so that it is not optimal for the auctioneer to provide the efficient level of information. We also show that as the number of participants in the auction increases both the efficient and the optimal level of information increase and both converge when the number of bidders goes to infinity.
\end{abstract}

Keywords: Auctions, Competition, Private Values, Optimal and Efficient Provision of Information.

JEL classification numbers: D44, D82, D83.

\footnotetext{
*Department of Economics and Business, Universitat Pompeu Fabra

${ }^{\dagger}$ Instituto de Análisis Ecónomico, CSIC. Part of this work was done while J-J Ganuza was visiting CEMFI. He thanks the hospitality of CEMFI as well as the Financial support of the Spanish Ministry of Science and Technology under project SEC2003-08080-C02-01. José Penalva acknowledges financial support from the Spanish Ministry of Science and Technology under project DGESIC P105-8002 and the Fundación BBVA. We also like to thank audiences at Gerzensee 2005, the ESSEM 2004, EARIE 2004, PET 2004, SED 2004, Games 2004, Simposio de Análisis Económico 2004, Barcelona Jocs 2004 for their questions and comments. We also like to thank Jean-Pierre Benoit, Roberto Burguet, Guillermo Caruana, Angel Hernando, Ian Jewitt, Jonathan Levin, David Levine, Marco Ottaviani and John Quah for their comments and suggestions. Corresponding author: juanjo.ganuza@upf.edu.
} 


\section{Introduction}

This paper studies the auctioneer's incentives to provide bidders with information about the object to be sold in a private values setting where the match between the characteristics of the object and the preferences of bidders plays a crucial role. In particular, we will be comparing the efficient and the auctioneer's optimal provision of information, and how these change as competition changes.

There are many situations in which the auctioneer can to some extent affect bidders' information. Take Internet auctions for example. In most of these auctions sellers have most of the information about the goods on sale and they decide how much information to reveal to bidders by posting electronic images, providing text descriptions, etc. Similarly, governments soliciting bids to execute a public project or a company selling a subsidiary have a lot of information on the goods at auction and control what type of information and in what way it will reach bidders.

The question of whether an auctioneer with information that is useful to bidders should keep it hidden or disclose it has a very powerful answer, the so called "linkage principle" derived by Milgrom and Weber (1982) in an affiliated values environment. According to the linkage principle the expected-revenue-maximizing policy for the auctioneer is to commit to fully and publicly announce all information he has. Thus, the level of information provided to the market is the efficient one and is independent of competition.

As we will demonstrate below, this result does not hold in private value auctions when the auctioneer reveals information about the object. The reason is that the effect of this type of information is fundamentally different in common value versus private value settings. In common value environments, bidders' preferences are aligned, bidders react symmetrically to the information revealed by the auctioneer and the 
revelation of information does not generate bidder rents. In private value environments bidders have heterogenous preferences, and their valuations depend on the match between their preferences and the characteristics of the object. This means that any information revealed by the auctioneer will be perceived differently by different bidders, raising the valuation of some bidders while reducing that of others. This asymmetric reaction to information leads to a more spread out distribution of bidders' expected valuations and may result in bidders' informational rents ${ }^{1}$.

We show that, when facing the decision of how much information to reveal in a private value setting, the auctioneer faces two opposing forces: more information improves the efficiency of the match while it also increases informational rents. As the auctioneer balances improved efficiency (which raises revenues) with increased informational rents (which reduce them), the auctioneer will reveal an inefficient amount of information ${ }^{2}$.

Further, we are interested in the relationship between the level of information in the market and competition. We prove that total surplus and the auctioneer's expected revenue are supermodular in the number of bidders and the level of information precision. This implies that the efficient and the auctioneer's optimal amount of information provision are increasing in the level of competition measured in terms of the number of bidders in the auction.

Total surplus, in our setting, is determined by the cost of information and the match between the object and the winning bidder's preferences. Adding an extra bidder increases the opportunity of a better match and the marginal value of information,

\footnotetext{
${ }^{1}$ Information of a different kind could have different implications. For example, in Milgrom and Weber (1982) the auctioneer reveals his valuation of the object. In a private value setting, where the auctioneer's valuation is independent of bidders' valuations, knowing the auctioneer's valuation has no effect on bidders' valuations and the linkage principle applies in a trivial sense.

${ }^{2} \mathrm{~A}$ related and complementary branch of the literature studies the bidders' private incentives to acquire information, see for example Matthews (1984), Tan (1992), Stegeman (1996), Persico (2001), Bergemann and Välimäki (2002), and Compte and Jehiel (2004).
} 
since this bidder reaps some efficiency gains from more information without reducing the gains of existing bidders. This makes information and competition complements when maximizing total surplus.

The auctioneer's optimal choice of information is more complex: expected revenue is total surplus minus informational rents of the winning bidder. With two bidders the negative effect of information, increased informational rents, overwhelms the gains from a better match, and the value of information is negative. As competition reduces informational rents, the compounded effect of competition on total surplus and informational rents is to increase the incentives of the auctioneer to provide more information. Eventually, the gains from the match topple the negative effect of informational rents and it becomes optimal for the auctioneer to provide information. Thereafter, the optimal amount of information provided by the auctioneer increases with the number of bidders. Finally, we show that as the number of participants goes to infinity informational rents disappear. Consequently total surplus and expected revenues converge and so do the optimal and the efficient levels of information.

Our results are demonstrated in the context of a standard private value auction. Prior to the auction we allow the auctioneer to provide bidders with information in the form of private signals correlated with their private valuations. The auctioneer controls the informativeness of the signals and chooses it so as to maximize his expected revenue from the auction ${ }^{3}$. We define a notion of informativeness, which we call precision, and which relates information to how spread out is the distribution of bidders' expected valuations. Once the auctioneer chooses the precision of the signals it becomes common knowledge, but the actual realizations of the private signals are known only by bidders. Each bidder uses this information (the level of precision and the realization of his

\footnotetext{
${ }^{3}$ Note that in our setup, the precision of information is known only when the auctioneer discloses the information. Eső and Szentes (2003) and Board (2004) study the case where the auctioneer can sell the information and extract bidders' future informational rents.
} 
private signal) to update his expected valuation of the object and then the auction takes place.

Note that we are modeling the match between the information on the object revealed by the auctioneer and privately known preferences implicitly, via private signals which are correlated with agent's true and unknown valuations. An alternative approach is to model this match explicitly, i.e. have the auctioneer provide a public signal related to the characteristics of the object and have agents with private information about their preferences interpret the signal and revise their expected valuations. Ganuza (2004) follows the direct approach in a Hotelling setting where an auctioneer sells a good to bidders who are located on a circle according to a uniform distribution. In this alternative symmetric setting, he finds the same tradeoff between efficiency and informational rents.

Bergemann and Pesendorfer (2003) study the design of the optimal auction and the optimal information structure in private value settings. They allow the auctioneer to provide information to bidders asymmetrically where we focus on the case where the auctioneer is constrained to provide information symmetrically ${ }^{4}$. Moreover, the information the auctioneer gives to bidders in Bergemann and Pesendorfer (2003) takes the form of partitions which are difficult to rank in terms of informational content as competition (the number of bidders) changes. Our approach is closer to the work of Bergemann and Välimäki (2002), Persico (2000), and Athey and Levin (2001). As in those papers we work with information structures that can be ordered in terms of their informativeness.

Board (2005) gives the auctioneer a dichotomous choice: provide no information

\footnotetext{
${ }^{4}$ Such a constraint arises naturally in many real problems: in internet and other auctions difficulties in identifying active bidders may make personalized information impractical; in government-related auctions legal restrictions often require the auctioneer to publicly release information and explicitly forbid asymmetric information provision in order to avoid favoritism or corruption; furthermore, sharing or leaking of information further undermine the desired effects of information discrimination.
} 
or reveal bidders' true valuations completely. Using convexity arguments he finds that the auctioneer will not reveal bidder valuations when there are only two bidders and that this revelation will occur only if the number of bidders is sufficiently high. Board (2005) provides an example of a family of distributions of bidder valuations such that the number of bidders necessary for full revelation can be made arbitrarily large. He also relates his results to the bundling decision in multi-unit auctions. Our paper explains in a general setting with partial and costly revelation of information that the basis for his results is a generic complementarity between information and competition.

Our paper is also related to the literature on providing information to consumers in imperfectly competitive markets, and in particular to Lewis and Sappington (1994) who focus on the case of monopoly. They examine whether the monopolist should allow the consumers to acquire information about their tastes for his product. Improved private information enables the monopolist to charge higher prices to high-value buyers, but can also provide rents to buyers. Moscarini and Ottaviani (2001) obtain similar results to Lewis and Sappington (1994) in an oligopolistic environment. They study a situation in which two sellers compete for a single buyer who observes a private signal on the relative quality of their goods. They find that sellers, like our auctioneer, may lose from the release of public information.

\section{The Model}

An auctioneer wants to sell an object he values at zero to one of $n$ (ex-ante) identical risk-neutral bidders (indexed by $i=1, \ldots, n$ ). Bidders' valuations of the object are private and unknown. Bidder $i$ 's realized valuation after the auction is described by a random variable, $V_{i}$. We assume all agents are ex-ante identical so that for all 
$i=1, \ldots, n, V_{i}$ is independently distributed on $\mathcal{V}=[0,1]$ according to a common distribution $H(v)=\operatorname{Pr}\left(V_{i} \leq v\right)$ with mean $\mu$.

The utility obtained by bidder $i$ from winning the auction is quasilinear. If the realized valuation is $v_{i}$ and he makes a monetary payment of $t_{i}$, the utility obtained is given by

$$
u_{i}\left(v_{i}, t_{i}\right)=v_{i}-t_{i}
$$

All bidders start with identical priors, described by $H$, and no other information on the object ${ }^{5}$. Hence, their expected valuations of the object will be the same and equal to $\mu$.

The auctioneer can reveal information on the object prior to the auction. The production of information is costly. By paying an amount $\delta \in[0, \infty)$ the auctioneer will generate information which bidders receive in the form of private signals $\left(X_{i}\right)_{i=1}^{n}$. Bidder $i$ receives the private signal $X_{i}$ and no other. The signals are independent and identically distributed random variables. We assume that these signals are drawn from the space of signals, $\mathcal{X} \subseteq \mathbf{R}$, and for each $i=1, \ldots, n$, each $X_{i}$ is informative only about bidder $i$ 's own true and unknown valuation of the object, $v_{i}$.

When the auctioneer decides how much to invest in providing information, $\delta$, he determines the informational content of the private signals, $\left(X_{i}\right)_{i=1}^{n}$. Formally, by choosing $\delta$, the auctioneer determines the information structure, where an information structure is a joint distribution, $\mathbf{F}_{\delta}$ over signals, $\left(X_{i}\right)_{i=1}^{n}$ and valuations $\left(V_{i}\right)_{i=1}^{n}$, indexed by $\delta$. As the signals are independent, there exists a distribution

\footnotetext{
${ }^{5}$ Bidders could start with private independent estimates of the value of the good. This would add a great deal of technical complexity that would complicate the demonstration of our results but as long as the ex-ante symmetry across bidders (from the point of view of the auctioneer) is preserved we do not see any reason why our results should change in any qualitatively significant way.
} 
$F_{\delta}(v, x)=\operatorname{Pr}(V \leq v, X \leq x)$, such that

$$
\mathbf{F}_{\delta}\left(V_{1} \leq v_{1}, \ldots, V_{n} \leq v_{n}, X_{1} \leq x_{1}, \ldots, X_{n} \leq x_{n}\right)=\prod_{i=1}^{n} F_{\delta}\left(v_{i}, x_{i}\right)
$$

We leave out the $i$ subscripts on signals and valuations whenever they are clear from the context. With minor abuse of notation let $F_{\delta}(x)$ and $F_{\delta}(v)$ denote the marginal distributions of $X$ and $V$ respectively. As priors have to be consistent with the joint distribution $F_{\delta}(v)=H(v)$.

We will be comparing the effect of different levels of investment on information and this requires that we make the signals generated by different information structures comparable. This is done by transforming the realized signal $X_{i}$ into a new random variable, $\Pi_{i}$, using the probability integral transformation: $\Pi_{i}=F_{\delta}\left(X_{i}\right)$. Then, $F_{\delta}^{-1}\left(\Pi_{i}\right)=X_{i}$, where $F_{\delta}^{-1}$ is the right-continuous inverse of the marginal distribution $F_{\delta}(x)$. We will assume that all random variables are non-trivial.

The new signal, $\Pi_{i}$, has exactly the same informational content as $X_{i}$ so that we can use $\Pi_{i}$ 's instead of $X_{i}$ 's unambiguously. More importantly, the marginal distribution of $\Pi_{i}$ has a very useful property: it is the uniform distribution on $[0,1]$ and independent of $\delta$. Let $F_{\delta}(\pi \mid v)$ and $F_{\delta}(v \mid \pi)$ be the conditional distributions, where $F_{\delta}(v \mid \pi)=\operatorname{Pr}(V \leq$ $\left.v \mid X=F_{\delta}^{-1}(\pi)\right)$.

We will not yet formalize exactly how a higher $\delta$ generates more informative signals, we discuss that in the following section, section 3. At this stage, it suffices to realize that the information structures are indexed by $\delta$, that this $\delta$ serves to rank the information structures by the informativeness of signals, and finally, that $\delta$ is public information to all bidders.

After the auctioneer has released the information, the awarding process takes place. To participate in this process, each bidder combines his knowledge of $\delta$ and the realization of the private signal, $\pi_{i}$, to update his expected valuation of the object $E\left[v_{i} \mid \pi_{i}, \delta\right]$ 
(also referred to as the interim valuation and denoted $W_{i}\left(\pi_{i}, \delta\right)$ ) using Bayes' rule. The auctioneer sells the object using a second-price sealed-bid auction. ${ }^{6}$ For simplicity we abstract from reserve prices and assume that the object is always sold. Summarizing, the model is structured as follows:

1. Bidders start with common priors over their unknown valuations for the object.

2. Prior to the auction, the auctioneer, knowing the number of bidders, $n$, decides how much to spend on information, $\delta$ (the more he spends the more informative will the signals be). This decision becomes public information.

3. Given $\delta$, each bidder receives a private signal $\pi_{i}$ over his valuation.

4. According to $\delta$ and the private signals, $\left(\pi_{i}\right)_{i=1}^{n}$, bidders update their valuations of the object.

5. The second-price sealed-bid auction takes place.

We now define and discuss what it means for the auctioneer to provide more information and present sufficient conditions for a family of information structures to be ordered in terms of the informativeness of the signals. Then the analysis will proceed in the usual way: we characterize the Perfect Bayesian Equilibrium starting from the auction and moving backwards, both for the efficient and auctioneer optimal cases.

\footnotetext{
${ }^{6}$ As will become apparent subsequently, the choice of the second price auction as the awarding mechanism is done without loss of generality as long as the conditions for the revenue equivalence theorem are present: bidders are symmetric in expected terms, risk neutral and their expected valuations of the object are independently distributed. Thus, all "standard" auctions (second-price sealed-bid, first-price sealed-bid, oral ascending (English) or oral descending (Dutch)) and many non-standard auctions such as an "all-pay" auction yield the same expected revenue to the auctioneer, bidders make the same expected payments as a function of their valuations and, as a consequence, the same results would be obtained.
} 


\section{Information Structures}

Given any $\delta>0$, signals are informative about valuations, $V$. We formalize this by assuming that given two signals $\pi^{\prime}$ and $\pi$, such that $\pi>\pi^{\prime}$, receiving the larger signal, $\pi$, is good news in the sense of Milgrom (1981); i.e. the posterior distribution of true valuations conditional on $\pi, F_{\delta}(v \mid \pi)$, dominates the posterior distribution of true valuations conditional on $\pi^{\prime}, F_{\delta}\left(v \mid \pi^{\prime}\right)$, in terms of First Order Stochastic Dominance (FOSD), denoted $F_{\delta}(\cdot \mid \pi) \geq_{s t} F_{\delta}\left(\cdot \mid \pi^{\prime}\right)$, (that is $F_{\delta}(v \mid \pi) \leq F_{\delta}\left(v \mid \pi^{\prime}\right)$ for all $\left.v \in \mathcal{V}\right)$. We assume that this condition holds for all distribution functions throughout the paper. This implies that agent's interim (expected) valuations, $W_{i}(\pi, \delta)$, are a nondecreasing function of the realization of the signal, $\pi$.

We want to formalize how a higher $\delta$ leads to more informative signals (better information on the characteristics of the object). The primary approach to defining the informativeness of a structure, $\mathbf{F}_{\delta}$, is based on the pioneering work of Blackwell. Blackwell(1951) considers the decision problem of an individual who has to choose an action based on the realization of the signal. He defines a signal structure, $\mathbf{F}_{\delta}$, to be more informative than another, $\mathbf{F}_{\delta^{\prime}}$, if every decision maker prefers $\mathbf{F}_{\delta}$ to $\mathbf{F}_{\delta^{\prime}}$, and shows it is equivalent to the statistical notion of sufficiency. Subsequently weaker notions of informativeness have been used (Lehmann(1988), Jewitt(1997), Athey Levin(2001)) which focus on when all decision makers in a particular class (e.g. those with supermodular payoff functions) prefer $\mathbf{F}_{\delta}$ to $\mathbf{F}_{\delta^{\prime}}$.

The problem we study requires a different approach to the notion of informativeness. The auctioneer will take no additional actions after chosing $\delta$ - the auction mechanism takes over ${ }^{7}$.

\footnotetext{
${ }^{7}$ Our approach contrasts with that of those who study information acquisition in auctions (Bergemann Välimäki(2002), Persico(2000)) and use Lehmann's notion of informativeness. In their settings, the individuals making the information decision (buyers) are the same agents who will be choosing bids in the auction based on the realization of the signal they receive.
} 
Let $\Pi$ be a random variable uniformly distributed on $[0,1]$. The random variable $W(\Pi, \delta)$ represents the bidders' updated expected valuations from the point of view of the auctioneer. For our type of problem, where the auctioneer takes no further actions after choosing the level of information, $\delta$, what matters is how $\delta$ affects the distribution function of $W(\Pi, \delta)$. By increasing the informativeness of the signal, the distribution of $W(\Pi, \delta)$ becomes more spread out ${ }^{8}$. This is because the increased importance of the realization of the signal and the corresponding reduction in the importance of the prior makes updated expected valuations more sensitive to the realized value of the signal. This sensitivity translates to greater variability of expected valuations and a more spread out distribution of $W(\Pi, \delta)$.

While all previous notions of informativeness lead to a more spread out distribution of $W(\Pi, \delta)$, our concept of informativeness is defined by requiring that this spreading out takes place in a very specific way. We will define more information based on the idea that more information makes the distribution of expected valuations more disperse:

Definition 1 (Bickel-Lehmann 1976) A random variable $X$ with cumulative distribution function $F$ is said to be more disperse than another random variable $Y$ with cumulative distribution function $G$, and denoted $X \geq_{\text {disp }} Y$ if for all $q, p \in[0,1], q>p$

$$
F^{-1}(q)-F^{-1}(p) \geq G^{-1}(q)-G^{-1}(p)
$$

Dispersion defines an ordering over distribution functions which implies further orderings of the distributions of their first and second order statistics, two crucial variables in our analysis ${ }^{9}$.

\footnotetext{
${ }^{8}$ One can also regard a more spread out distribution of updated expected valuations as more risky. The relation between risk and information is well-known, see Baker(2004) for some recent results relating these two concepts.

${ }^{9}$ For an overview of results relating dispersion and order statistics see Shaked and Shantiku$\operatorname{mar}(1994)$.
} 
Using dispersion, we will assume that $\delta$ orders information structures in terms of the precision (informativeness) of the signals, where greater precision is defined as follows:

Definition 2 The information structure $\mathbf{F}_{\delta}$ has more precise signals than $\mathbf{F}_{\delta^{\prime}}$ iff $\forall \pi, \pi^{\prime} \in[0,1], \pi>\pi^{\prime}$

$$
W(\pi, \delta)-W\left(\pi^{\prime}, \delta\right) \geq W\left(\pi, \delta^{\prime}\right)-W\left(\pi^{\prime}, \delta^{\prime}\right)
$$

Alternatively, our definition can be viewed as requiring that $W(\pi, \delta)$ be supermodular in $\pi$ and $\delta$, i.e. $W(\pi, \delta)-W\left(\pi^{\prime}, \delta\right)$ is increasing in $\delta$ or (equivalently) $W(\pi, \delta)-W\left(\pi, \delta^{\prime}\right)$ is increasing in $\pi$.

The intuition is that increasing precision increases the slope of the expected valuation function (as depicted on Figure 1).

When comparing two signals from the same information structure, we said that a higher signal is better news than a low signal because it raises bidder's expected valuation. As $W(\pi, \delta)$ and $W\left(\pi, \delta^{\prime}\right) \operatorname{cross}^{10}$, we can think of increasing precision as making low signals worse news and high signals better news.

Economists are more familiar with a different and yet related notion of 'spread out' distributions: Second Order Stochastic Dominance ${ }^{11}$ (SOSD). Dispersion is a stronger condition than SOSD:

Remark 1 Let $\mathbf{F}_{\delta}$ be more precise than $\mathbf{F}_{\delta^{\prime}}$, then $W\left(\Pi_{i}, \delta\right)$ is dominated by $W\left(\Pi_{i}^{\prime}, \delta^{\prime}\right)$ in the sense of SOSD.

To illustrate our concept we look at two basic families of information structures that are ordered according to precision.

\footnotetext{
${ }^{10}$ The law of iterated expectations implies $E[W(\Pi, \delta)]=E\left[W\left(\Pi, \delta^{\prime}\right)\right]=\mu$, so that if $W(\pi, \delta)$ and $W\left(\pi, \delta^{\prime}\right)$ were continuous and differentiable they would cross at a single point (as in Figure 1). In general, $W(\pi, \delta)-W\left(\pi, \delta^{\prime}\right)$ is monotone increasing and changes sign once.

${ }^{11}$ We have included the formal definition for those not familiar with it in Appendix A.1.
} 


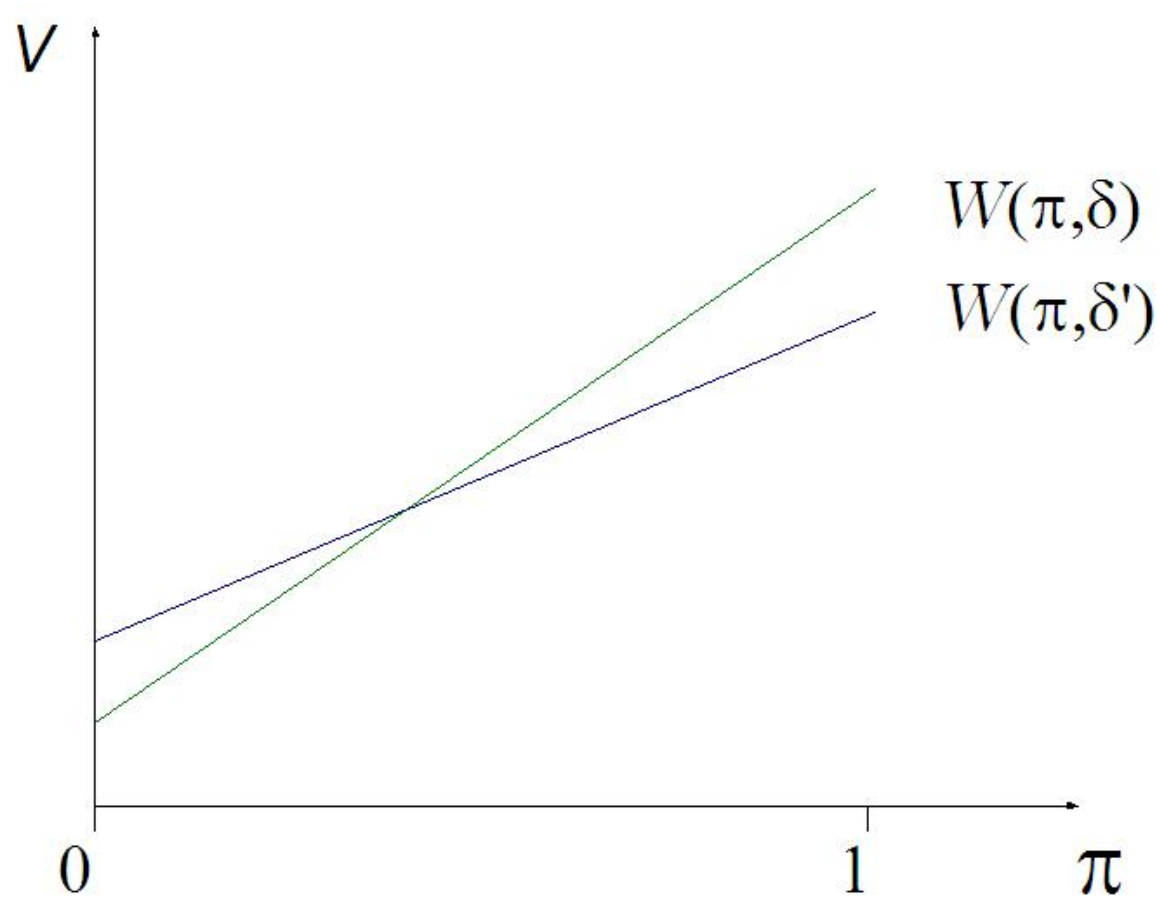

Figure 1: Precision and expected valuation functions

Example 1: Let $V$ be normally distributed with mean $\mu$ and variance $\sigma_{v}^{2}$, and let the distribution of the signal, $X$, conditional on the true valuation, $v$, be the realization of the valuation $v$ plus a noise terms, $\epsilon_{\delta}$, which is normally distributed with mean zero and variance $\sigma_{\delta}^{2}$. The variance of the noise term orders these information structures in terms of precision in the usual way: lower variance in the noise leads to greater precision of the signals.

Example 2: (Banerjee (1992)) Let $V$ be distributed according to the prior distribution $H(v)$ with mean $\mu$. The signal, $X$, represents the bidder's valuation $V$, but the signal reveals the truth, i.e. $v=x$, with probability $\delta$ and with the complementary probability the signal is false. We assume that when the signal is false it is pure noise, distributed according to the prior, $H(v)$, and independently of $v$. Thus, independently 
of the precision of the signal, $X$ is distributed according to $H(v)$. An increase in the probability that the signal reveals the truth, $\delta$, increases precision.

In Section 5 we will explore our notion of informativeness in greater detail, provide a sufficient condition for greater precision based on the properties of the joint distribution function, $F_{\delta}$, and relate precision to existing notions of informativeness.

\section{Releasing Information}

In this section, we want to contrast the optimal level of information provided by the auctioneer with the efficient level of information. We start by characterizing efficiency.

\subsection{The Efficient Release of Information}

The efficient level of information is that which maximizes the total surplus at the time of the information release. In our setup, total surplus is defined as the sum of the auctioneer's revenue and the interim utility of the bidder with the highest expected valuation at the time of the auction. As the price paid for the object is a pure transfer from the auctioneer to the winning bidder, total surplus is the expected valuation of the object by the winning bidder minus the cost of providing information. We first focus on expected valuation of the winning bidder.

Denote the highest realization of the signal by $x_{1}$, so that $\pi_{1} \equiv F_{\delta}\left(x_{1}\right)$. The winner of the auction will be the bidder receiving $\pi_{1}$ so that his expected valuation is: $V_{1}(n, \delta)=E\left[W\left(\Pi_{1}, \delta\right)\right]$ - the notation makes explicit the number of bidders as we shall be studying the effect of changing $n$. Let $U_{1: n}(p)$ be the cumulative distribution function of the first order statistic of $n$ independent uniform random variables on $[0,1]$. As the transformed signals $\Pi_{1}$ are independent and uniformly distributed on $[0,1]$,

$$
V_{1}(n, \delta)=\int_{0}^{1} W(p, \delta) \mathrm{d} U_{1: n}(p)
$$


As the auctioneer increases the level of information and, correspondingly, the precision of the signals, this expectation increases:

Proposition 1 The expected valuation of the winning bidder $V_{1}(n, \delta)$ is nondecreasing in $\delta$, the precision of the signals.

As increasing precision leads to giving greater weight to the signal realization relative to the prior, then more precision increases the expected valuation of the winner. Our model is designed to capture the situation where the auctioneer provides information about the features of the object and bidders have private information over their own preferences. Under this interpretation Proposition 1 says that providing more information improves the matching between the features of the object and the preferences of the winning bidder.

Thus, the trade-off faced when deciding the efficient amount to spend on information, $\delta^{E}$, is between increasing the expected valuation of the winning bidder and increasing the cost of providing that information to the market:

$$
\delta^{E} \in \underset{\delta}{\operatorname{argmax}} V_{1}(n, \delta)-\delta
$$

The next proposition states the relationship between the efficient level of precision and the level of competition.

Proposition 2 The total surplus is supermodular in $\delta$ and $n$.

Proposition 2 states that the difference in terms of expected surplus between two levels of signal precision is larger the larger the number of bidders. From an economic point of view, the larger the number of bidders, the larger the value of information. The intuition is that having more draws from the pool of bidder preferences increases the incentives of the auctioneer to improve the matching between the object and bidder preferences by increasing the amount of information. 
Corollary 1 The efficient level of precision, $\delta^{E}$, is nondecreasing in the number of bidders.

As the number of bidders in the auction increases the marginal effect of adding information is greater so that with more bidders it is efficient to spend more on information.

\subsection{The Auctioneer's Optimal Information Release}

Having characterized the efficient level of information, we now turn to the auctioneer's problem: how much information to release in order to maximize expected revenue from the auction.

We first characterize the bidder's optimal strategy given $\delta$ and the realization of his private signal $x_{i}$. Let $x_{2}$ denote the second-highest signal which, in the second-price auction, is the price the auctioneer receives for the object. Using the transformed variables, $\pi_{2} \equiv F_{\delta}^{-1}\left(x_{2}\right)$. As the transformed signals are independent and uniformly distributed on $[0,1]$, the cumulative distribution of the second order statistic in a sample of $n$ iid random variables uniformly distributed on $[0,1], U_{2: n}(p)$, is the cumulative distribution function of $\Pi_{2}$. Then the expected price in the auction (the expected valuation of the bidder with the second highest signal) is:

$$
V_{2}(n, \delta)=\int_{0}^{1} W(p, \delta) \mathrm{d} U_{2: n}(p)
$$

The next proposition states the relationship between expected price and the amount of information.

Proposition 3 Suppose $\delta>\delta^{\prime}$. (i) For $n=2$ information has a negative value: $V_{2}(2, \delta) \leq V_{2}\left(2, \delta^{\prime}\right)$. On the other hand, (ii) there exists $n^{\prime}$ such that for all $n>n^{\prime}$ information is valuable: $V_{2}(n, \delta) \geq V_{2}\left(n, \delta^{\prime}\right)$. 
While Proposition 1 shows that information always improves efficiency, Proposition 3 states that the relationship between expected price and information is less clear cut and depends on the level of competition as measured by the number of bidders in the auction. In fact, the first part of Proposition 3 points out that if the number of bidders is small enough, information can reduce the auctioneer's revenue. The driving force behind this result is that information, by spreading out the distribution of expected valuations, makes bidders more heterogeneous. This heterogeneity plus a lack of competition generates informational rents which reduce auctioneer revenue and counters the positive effect of information, that the sale is more efficient. The technical intuition is also straight-forward: when there are only two bidders then on average the signal of the loser in the auction (and hence his expected valuation and the price) will be below the mean of the distribution. Thus, it is easy to see that providing information is worse for the auctioneer than not providing any information at all. On the other hand, it seems intuitive that as competition increases, informational rents fall and information will become valuable eventually (as stated in the Proposition).

The next proposition addresses the question of how the rents generated by greater precision are distributed between the auctioneer and the winner in the auction. Given an information structure with precision $\delta$, the expected informational rents of the winning bidder is the difference between his valuation and the valuation of the second closest bidder (which is the price paid for the object)

$$
R_{w}(n, \delta)=V_{1}(n, \delta)-V_{2}(n, \delta)
$$

Proposition 4 The expected informational rents of the winning bidder are nondecreasing in $\delta$.

Proposition 4 is linked to the fact that more information makes the distribution 
of interim valuations more disperse. Hence Proposition 4 shows us the drawback of providing information to the market: it increases bidders' rents.

To establish the auctioneer's optimal strategy, we have to characterize the level of information, $\delta^{A}$, that maximizes the difference between the expected price and the cost of providing more information:

$$
\delta^{A} \in \underset{\delta}{\operatorname{argmax}} V_{2}(n, \delta)-\delta
$$

By comparing (1) and (2) it is easy to see that the structure of the auctioneer's problem is identical to that of total surplus maximization with $V_{1}(n, \delta)$ substituted by $V_{2}(n, \delta)$, so that the intuition behind the results presented in the following Proposition and its Corollary is similar as those for Proposition 2 and Corollary 1.

Proposition 5 The auctioneer's expected profits are supermodular in $\delta$ and $n$.

Hence the larger the number of bidders, the larger the incentives of the auctioneer to provide information.

Corollary 2 The optimal level of signal precision, $\delta^{A}$, is nondecreasing in the number of bidders, $n$.

Finally, the following presents the main result of the paper.

Theorem 1 The optimal level of signal precision is lower than the efficient level: $\delta^{A} \leq$ $\delta^{E}$. The difference between the efficient and the optimal level of precision converges to 0 as the number of bidders goes to infinity.

To better understand the result for the case where the value of information is not negative for the auctioneer one can rewrite the auctioneer's problem as:

$$
\delta^{A} \in \underset{\delta}{\operatorname{argmax}} V_{1}(n, \delta)-\delta-R_{w}(n, \delta)
$$


This formulation clarifies the trade-off faced by the auctioneer when providing information to the market. On the one hand, when the auctioneer provides more information, the efficiency of the auction process goes up $\left(V_{1}(n, \delta)\right.$ is nondecreasing in $\delta$ - Proposition 1). On the other hand, the increase in information also raises the informational rents of the winning bidder $\left(R_{w}(n, \delta)\right.$ is nondecreasing in $\delta$-Proposition 4). The optimal balance of these two opposing effects leads the auctioneer to provide less information than would be efficient. In other words, the auctioneer will restrict the information released to the market in order to make bidders more homogeneous, with the underlying goal of intensifying competition and increasing his expected revenue. As

the number of bidders increases, the informational rents are reduced and the trade-off is weakened. In the limit, as the number of bidders goes to infinity, the informational rents disappear and with it the difference between efficient and optimal information release.

\section{Precision and other Notions of Informativeness}

In this section we will provide a sufficient condition for greater signal precision based on the properties of the joint distribution functions. We will also consider alternative notions of informativeness based on the properties of the distribution of expected valuations, compare these notions of informativeness with standard ones and discuss how these relate to previous results in the paper.

\subsection{A Sufficient Condition For Greater Precision}

Signal precision is based on the shape of the distribution of updated expected valuations. In some cases this condition can be verified directly but at times, such as when looking at parameterized families of bivariate distributions, one might prefer to use an informativeness condition based on some property of the joint distribution function. 
From a statistical point of view an information structure in which signals are informative about valuations is one in which larger realizations of the signal are probabilistically associated with higher valuations, so that signals and valuations are positively dependent.

By changing $\delta$, the auctioneer changes the joint distribution of signals and valuations, $F_{\delta}(v, \pi)$. As a signal is informative because "larger realizations of the signal are probabilistically associated with higher valuations" then, by increasing the informativeness of the signals we should be increasing the probabilistic relationship between signals and valuations. In other words, increasing informativeness makes signals and valuations more positively dependent.

The following defines an ordering over joint distribution functions in terms of positive dependence:

Definition 3 (Caperaa and Genest 1990) Let $F_{\delta}$ and $F_{\delta^{\prime}}$ be two joint distribution functions with posterior distribution functions $F_{\delta}(v \mid \pi)$ and, $F_{\delta^{\prime}}(v \mid \pi)$. The distribution function $F_{\delta}$ is more stochastically increasing in $\pi$ than $F_{\delta^{\prime}}$ if the function:

$$
\gamma(v, \pi)=F_{\delta}^{-1}\left(F_{\delta^{\prime}}(v \mid \pi) \mid \pi\right) \quad \text { is nondecreasing in } \pi .
$$

If $F_{\delta}(v \mid \pi)$ and $F_{\delta^{\prime}}(v \mid \pi)$ are continuous in $v$ for all $\pi$ then $\gamma(v, \pi)$ is increasing in $\pi$ and is equivalent ${ }^{12}$ to the following single-crossing condition: for all $\pi^{\prime}<\pi, v, v^{\prime} \in \mathcal{V}$,

$$
F_{\delta^{\prime}}\left(v \mid \pi^{\prime}\right) \geq F_{\delta}\left(v^{\prime} \mid \pi^{\prime}\right) \Rightarrow F_{\delta^{\prime}}(v \mid \pi) \geq F_{\delta}\left(v^{\prime} \mid \pi\right)
$$

Those familiar with the literature on information economics will recognize that Condition (3) is Lehmann (1988)'s notion of effectiveness but with valuations and signals interchanged. Furthermore, if $F_{\delta}(v \mid \pi)$ is differentiable in $\delta$ and $v$ then greater

\footnotetext{
${ }^{12}$ See Fang and Joe(1992).
} 
precision is also equivalent to the Spence-Mirrlees type condition ${ }^{13}$ :

$$
\frac{\partial F_{\delta}(v \mid \pi) / \partial \delta}{\partial F_{\delta}(v \mid \pi) / \partial v} \text { is nondecreasing in } \pi \text {. }
$$

Proposition 6 Suppose $F_{\delta}(v \mid \pi)$ and $F_{\delta^{\prime}}(v \mid \pi)$ are differentiable in $\delta$ and $v$. If $F_{\delta}$ is more stochastically increasing in $\pi$ than $F_{\delta^{\prime}}$ then $\mathbf{F}_{\delta}$ has more precise signals than $\mathbf{F}_{\delta^{\prime}}$.

Summarizing, condition (3) can be interpreted as a mirror image of Lehmann's effectiveness condition and is sufficient to ensure that two information structures are ordered in terms of the precision of their signals.

\subsection{Informativeness and Ex-post Valuations}

Our approach is based on the basic intuition that in a private value setting, more information about the good (increasing $\delta$ ) makes $W(\Pi, \delta)$ more spread out, more variable. We have formalized this idea by requiring that $W(\Pi, \delta)$ be more disperse than $W\left(\Pi, \delta^{\prime}\right)$. We consider two other conditions based on similar concepts that have been used to compare random variables in terms of their variability: a single-crossing condition (related to the idea of 'more dangerous'14 random variables), and an integral condition that is related to the usual second order stochastic dominance. Let $\delta>\delta^{\prime}$ and $\pi>\pi^{\prime}$ :

- Dispersion: $W(\pi, \delta)-W\left(\pi, \delta^{\prime}\right) \geq W\left(\pi^{\prime}, \delta\right)-W\left(\pi^{\prime}, \delta^{\prime}\right)$.

- SC-dispersion: $W\left(\pi^{\prime}, \delta\right)-W\left(\pi^{\prime}, \delta^{\prime}\right) \geq 0$ implies $W(\pi, \delta)-W\left(\pi, \delta^{\prime}\right) \geq 0$.

- Integral-dispersion: $\int_{0}^{\pi}\left(W(p, \delta)-W\left(p, \delta^{\prime}\right)\right) d p \leq 0$ for $\pi$ and $\pi^{\prime}$.

\footnotetext{
${ }^{13}$ Jewitt (1997) gives a detailed comparison of different notions of informativeness and illustrates the connection between the standard Spence-Mirrlees condition and Lehmann's notion of effectiveness.

${ }^{14}$ The nomenclature is from actuarial science (where the notion was introduced by Ohlin (1969)). This concept appears earlier in Karlin and Novikoff (1963).
} 
These notions of dispersion, when applied to the distribution of expected valuations from two information structures can be used to define different notions of informativeness. In the same way as we have used the dispersion condition to define more precise signals we can use SC-dispersion and integral-dispersion to define more SC-precise and integral-precise signals. Note that precision implies SC-precision, which itself implies integral-precision.

\subsection{Variability and Information}

Standard notions of information are based on the value of information for an individual decision maker (Blackwell (1951), Lehmann (1988), Jewitt (1997), Athey and Levin (2001)). Athey and Levin (2001) present necessary and sufficient conditions for all decision makers with different classes of payoff functions to prefer one information structure over another. If we restrict attention to the class of decision makers with nondecreasing incremental returns, the necessary and sufficient condition is denoted the MIO-ND condition ${ }^{15}$. Given that the other notions are sufficient for information to be valuable for all decision makers with nondecreasing incremental returns, MIOND is the weakest notion of informativeness, and as Blackwell's applies to all decision makers it is the strongest.

We can now relate these notions of information with the conditions stated above.

Proposition 7 (i) Blackwell's notion of information does not imply signals are more SC-precise and (ii) MIO-ND implies signals are more integral-precise.

Hence, none of the standard notions of informativeness imply SC-precision (or precision) and all standard notions of informativeness imply integral-precision.

\footnotetext{
${ }^{15}$ The definition of MIO-ND is reproduced in the Appendix.
} 


\subsection{Weakening Informativeness and PV Auctions}

If we consider that information provision is costly, then we know from Milgrom and Shannon (1994) that we need supermodularity of the functions $V_{1}(\delta, n)$ and $V_{2}(\delta, n)$ for the comparative results relating information and competition. Among the three information conditions discussed above (precision, SC-precision and integral-precision) only the strongest one, precision, ensures the supermodularity of $V_{1}(n, \delta)$ and $V_{2}(n, \delta)$.

However some comparative static results can be obtained using SC-precision and integral-precision in a framework where the provision of information is costless.

If $\delta$ only implies signals are more integral-precise (and not more precise) Proposition 1 holds. Hence, in a setup with costless provision of information the efficient policy is full information disclosure. Proposition 3 and Theorem 1 also hold, i.e. if the number of bidders is small information has a negative value, the auctioneer will optimally follow a full disclosure strategy if there is enough competition.

The notion of SC-precision adds on integral-precision by ensuring that $V_{2}(\delta, n)$ is single-crossing so that for all $n$, the optimal amount of information provided by the auctioneer is weakly monotonic in the number of bidders.

\section{Conclusions}

We were interested in what happens when the auctioneer can release information to bidders and how the amount of information released relates to the level of competition in private value auctions. We have set up a standard auction model and used a general notion of informativeness to study this question. We have shown that the optimal level of information released by the auctioneer is not the same as the efficient level, which contrasts with the "linkage principle". This is because in private value settings, there are two factors that determine the optimal provision of informa- 
tion: (i) improved information increases the efficiency of the auction; (ii) improved information also increases the informational rents of the winning bidder. These two effects represent opposing forces for the auctioneer: improved efficiency raises revenues while increased informational rents reduce revenues. Hence, the optimal amount of information released is below the efficient one.

Our second main result relates competition and the information provided to the market. We show that there is a complementarity between competition and information when maximizing total welfare and also, when maximizing auctioneer revenues. Then, both the efficient and the optimal level increase with the number of bidders. We conclude by showing that as the number of bidders goes to infinity, the difference between the efficient and optimal solutions vanishes.

The complementarity between information and competition opens up a number of interesting avenues for future research. For example, this could be an ingredient in explaining the prevalence of incomplete contracting in many real life situations. In procurement, one can consider the possibility of reducing the degree of specificity in a contract in order to homogenize the market and inject an additional degree of competition into the procurement process.

Another possible extension is to consider endogenising the degree of competition in the auction by adding an initial stage whereby firms/bidders decide whether to enter into the auction or not. The complementarity between the level of information provision and competition should intuitively lead to multiplicity of equilibria in the entry game. There will be equilibria with low competition and a low level of information and other equilibria with lots of competition and a high level of information ${ }^{16}$.

It would also be interesting to pursue the empirical content of our results. Along

\footnotetext{
${ }^{16}$ This and other extensions, such as introducing a reserve price are studied using a parameterized family of information structures in Ganuza and Penalva (2004).
} 
theses lines, Kavajecz and Keim (2004) document how some institutional investors use 'blind auctions' to allocate orders to buy and sell large number of shares to brokers. In these auctions the auctioneer (the institutional investor) aggregates a large number of different types of orders into a single package and consciously provides relatively little detail on the shares in the package to brokers. Also, brokers' (bidders') valuations of the package have a substantial private value component (the value of the package to a broker strongly depends on how the package of shares matches the demand from his other clients and which varies substantially from broker to broker). These types of auctions would illustrate our claim that the auctioneer withholds information on the shares in the package in order to make the auction more competitive although it would be more efficient to provide all information available. 


\section{A Appendix}

\section{A.1 Preliminary Result and Notation}

Using the notation developed in the text, we will make repeated use of the following very well-known result, which we state as a lemma: if $X \geq_{s t} Y$ then for all nondecreasing functions $\psi, E[\psi(X)] \geq E[\psi(Y)]$.

Lemma 1 Let $X$ and $Y$ be real-valued random variables with cumulative distribution functions $F$ and $G$ respectively, such that $F(z) \leq G(z)$ for all $z \in \mathbf{R}$. For all bounded real-valued nondecreasing functions $\psi: \mathbf{R} \rightarrow \mathbf{R}$,

$$
\int_{\mathbf{R}} \psi(z) \mathrm{d} F(z) \geq \int_{\mathbf{R}} \psi(z) \mathrm{d} G(z)
$$

We also use the following notation: $U_{i: j}(x)$ is the cumulative distribution function (cdf) of a random variable $Y$ such that $U_{i: j}(x)=\operatorname{Pr}(Y \leq x)$. This random variable is the $i$ th order statistic from a sample of $j$ independently and identically uniform distributed random variables over $[0,1]$, where $U_{1: j}$ refers to the cdf of the maximum of the sample, $U_{2: j}$ to the cdf of the second highest realization in the sample and so on until $U_{j: j}$ which is the cdf of the minimum realization in the sample. We will also make use of the functional form of $U_{1: n}, U_{1: n}(\pi)=\pi^{n}, \pi \in[0,1]$.

Definition 4 (SOSD) A random variable $X$ with cumulative distribution function $F$, finite mean and support on $A \subseteq \mathbf{R}$ is dominated in terms of second order stochastic dominance $(S O S D)$ by a random variable $Y$ with cumulative distribution function $G$, finite mean and support on $B \subseteq \mathbf{R}$, denoted $X \leq_{S S D} Y$, if the expected value of $X$ is the same as that of $Y$ and for all $z \in \mathbf{R}$

$$
\int_{-\infty}^{z} F(x) \mathrm{d} x \geq \int_{-\infty}^{z} G(x) \mathrm{d} x .
$$




\section{Proof of Remark 1:}

Applying the law of iterated expectations: $\mu=E[E[v \mid X]]=E[W(\Pi, \delta)]$ and $\mu=E\left[E\left[v \mid X^{\prime}\right]\right]=E\left[W\left(\Pi^{\prime}, \delta^{\prime}\right)\right]$. If $X$ and $Y$, two random variables, have the same mean and $X$ is more disperse than $Y$, then $X$ is dominated by $Y$ in terms of SOSD (Shaked and Shantikumar (1994), Theorem 2.B.10).

\section{A.2 Proofs}

Proof of Proposition 1: We want to show that if $\delta>\delta^{\prime}$ then $V_{1}(n, \delta) \geq V_{1}\left(n, \delta^{\prime}\right)$.

This is equivalent to showing

$$
\int_{0}^{1}\left(W(\pi, \delta)-W\left(\pi, \delta^{\prime}\right)\right) \mathrm{d} U_{1: n}(\pi) \geq 0
$$

By the law of iterated expectations, the expected valuation of the distribution of expected valuations, $E[W(\pi, \delta)]=\mu$, must not depend on the information structure. Let $U_{1: 1}(\pi)=\pi$ denote the cumulative distribution function of the uniform. We can now write

$$
\int_{0}^{1}\left(W(\pi, \delta)-W\left(\pi, \delta^{\prime}\right)\right) \mathrm{d} \pi=\int_{0}^{1}\left(W(\pi, \delta)-W\left(\pi, \delta^{\prime}\right)\right) \mathrm{d} U_{1: 1}(\pi)=0
$$

Define the function $\psi(\pi) \equiv\left(W(\pi, \delta)-W\left(\pi, \delta^{\prime}\right)\right)$. By the definition of precision, $\psi(\pi)$ is nondecreasing in $\pi$. As $U_{1: n}(\pi)=\pi^{n} \leq U_{1: 1}(\pi)$ for all $n \geq 1$ and $\pi \in[0,1]$ and $\psi(\pi)$ is nondecreasing, we can apply Lemma 1 and the result follows.

Proof of Proposition 2: It suffices to show that $V_{1}(n+1, \delta)-V_{1}(n, \delta) \geq V_{1}(n+$ $\left.1, \delta^{\prime}\right)-V_{1}\left(n, \delta^{\prime}\right)$.

This is equivalent to showing

$$
\begin{aligned}
V_{1}(n+1, \delta)-V_{1}\left(n+1, \delta^{\prime}\right) & \geq V_{1}(n, \delta)-V_{1}\left(n, \delta^{\prime}\right) \\
\Leftrightarrow \int_{0}^{1}\left(W\left(\pi_{1}, \delta\right)-W\left(\pi_{1}, \delta^{\prime}\right)\right) \mathrm{d} U_{1: n+1}\left(\pi_{1}\right) & \geq \int_{0}^{1}\left(W\left(\pi_{1}, \delta\right)-W\left(\pi_{1}, \delta^{\prime}\right)\right) \mathrm{d} U_{1: n}\left(\pi_{1}\right)
\end{aligned}
$$


As $W(\pi, \delta)-W\left(\pi, \delta^{\prime}\right)$ is nondecreasing in $\pi$ and $U_{1: n+1}(\pi)=\pi^{n+1} \leq U_{1: n}(\pi)=\pi^{n}$ for all $\pi \in[0,1]$, we can apply Lemma 1 .

Proof of Corollary 1: Immediate from the results of Milgrom and Shannon (1994) and Proposition 2.

\section{Proof of Proposition 3:}

Part (i): We want to show that if $\delta>\delta^{\prime}$ then $V_{2}(2, \delta) \leq V_{2}\left(2, \delta^{\prime}\right)$. With two bidders and prior to their receiving their private signals, both have the same probability $\left(\frac{1}{2}\right)$ of being the bidder with the highest valuation. By the law of iterated expectations, the ex ante (before receiving the signal) expected valuation of bidders must not depend on the information structure. We can conclude that

$$
\frac{V_{1}(2, \delta)+V_{2}(2, \delta)}{2}=\frac{V_{1}\left(2, \delta^{\prime}\right)+V_{2}\left(2, \delta^{\prime}\right)}{2}=\mu
$$

Hence, $V_{1}(2, \delta)-V_{1}\left(2, \delta^{\prime}\right)=-\left(V_{2}(2, \delta)-V_{1}\left(2, \delta^{\prime}\right)\right)$. From Proposition $1, V_{1}(2, \delta)-$ $V_{1}\left(2, \delta^{\prime}\right) \leq 0$, which implies $V_{1}(2, \delta)-V_{1}\left(2, \delta^{\prime}\right) \geq 0$.

Part (ii): We want to show that there exists $n^{\prime}$ such that for $n \geq n^{\prime}, V_{2}(n, \delta)-$ $V_{2}\left(n, \delta^{\prime}\right) \geq 0$. It suffices to show first that for all $n, V_{2}(n, \delta)-V_{2}\left(n, \delta^{\prime}\right)$ is nondecreasing in $n$ and then to demonstrate that $\lim _{n \rightarrow \infty} V_{2}(n, \delta)-V_{2}\left(n, \delta^{\prime}\right)>0$.

To show $V_{2}(n, \delta)-V_{2}\left(n, \delta^{\prime}\right)$ is nondecreasing in $n$ for all $n$ we follow the same logic as in the proof of Proposition 2:

$$
\begin{aligned}
V_{2}(n+1, \delta)-V_{2}\left(n+1, \delta^{\prime}\right) & \geq V_{2}(n, \delta)-V_{2}\left(n, \delta^{\prime}\right) \\
\Leftrightarrow \int_{0}^{1}\left(W(\pi, \delta)-W\left(\pi, \delta^{\prime}\right)\right) \mathrm{d} U_{2: n+1}(\pi) & \geq \int_{0}^{1}\left(W(\pi, \delta)-W\left(\pi, \delta^{\prime}\right)\right) \mathrm{d} U_{2: n}(\pi)
\end{aligned}
$$

As $W(\pi, \delta)-W\left(\pi, \delta^{\prime}\right)$ is nondecreasing in $\pi$ and $U_{2: n+1}(\pi) \leq U_{2: n}(\pi)$ for all $\pi \in[0,1]$, we can apply Lemma 1. 
Finally, as $n$ goes to infinity, $U_{2: n}$ converges to a distribution with mass only at $\pi=1$. Thus,

$$
V_{2}(\delta, n)-V_{2}\left(\delta^{\prime}, n\right)=\int_{0}^{1} \psi(\pi) \mathrm{d} U_{2: n}(\pi)
$$

converges to $\psi(1)>0$.

\section{Proof of Proposition 4:}

We want to show that for $\delta>\delta^{\prime}, R_{w}(n, \delta) \geq R_{w}\left(n, \delta^{\prime}\right)$, i.e.

$$
V_{1}(n, \delta)-V_{2}(n, \delta) \geq V_{1}\left(n, \delta^{\prime}\right)-V_{2}\left(n, \delta^{\prime}\right)
$$

This is equivalent to

$$
V_{1}(n, \delta)-V_{1}\left(n, \delta^{\prime}\right) \geq V_{2}(n, \delta)-V_{2}\left(n, \delta^{\prime}\right)
$$

i.e.,

$$
\int_{0}^{1}\left(W\left(\pi_{1}, \delta\right)-W\left(\pi_{1}, \delta^{\prime}\right)\right) \mathrm{d} U_{1: n}\left(\pi_{1}\right) \geq \int_{0}^{1}\left(W\left(\pi_{2}, \delta\right)-W\left(\pi_{2}, \delta^{\prime}\right)\right) \mathrm{d} U_{2: n}\left(\pi_{2}\right)
$$

Again, using $W(\pi, \delta)-W\left(\pi, \delta^{\prime}\right)$ is nondecreasing in $\pi$ and the stochastic dominace of the first order statistic over the second, $U_{1: n}(\pi) \leq U_{2: n}(\pi)$ for all $\pi \in[0,1]$. Applying Lemma 1 concludes the proof.

Proof of Proposition 5: As in the proof of Proposition 2, it suffices to show that $V_{2}(n+1, \delta)-V_{2}(n, \delta) \geq V_{2}\left(n+1, \delta^{\prime}\right)-V_{2}\left(n, \delta^{\prime}\right)$, and we have demonstrated this in the proof of Proposition 3, part (ii).

Proof of Corollary 2: Immediate from the results of Milgrom and Shannon (1994) and Proposition 5.

Proof of Theorem 1: The auctioneer's problem is

$$
\delta^{A} \in \underset{\delta^{A}}{\operatorname{argmax}}\left\{V_{2}(n, \delta)-\delta\right\}
$$


This problem is equivalent to

$$
\delta^{A} \in \underset{\delta^{A}}{\operatorname{argmax}}\left\{V_{1}(n, \delta)-\delta-R_{w}(n, \delta)\right\}
$$

where $R_{w}(\delta)$ is as defined in the text.

Compare the formulation of the auctioneer's problem to the formulation of the social welfare maximization problem (equation 1 ). As $\delta^{A}$ solves the auctioneer's optimization problem

$$
E\left[W\left(\Pi_{1}, \delta^{A}\right)-\delta^{A}-R_{w}\left(\delta^{A}\right)\right] \geq E\left[W\left(\Pi_{1}, \delta^{E}\right)-\delta^{E}-R_{w}\left(\delta^{E}\right)\right]
$$

$R_{w}(\delta)$ is nondecreasing (Proposition 4) so that if $\delta^{A}>\delta^{E}$, then this last equation would imply

$$
E\left[W\left(\Pi_{1}, \delta^{A}\right)-\delta^{A}\right] \geq E\left[W\left(\Pi_{1}, \delta^{E}\right)-\delta^{E}\right]
$$

but this contradicts the fact that $\delta^{E}$ maximizes social surplus, so that $\delta^{A} \leq \delta^{E}$.

To establish the second part of the Theorem, consider the informational rents, $R_{w}(n, \delta)$

$$
\begin{aligned}
R_{w}(n, \delta) & =V_{1}(n, \delta)-V_{2}(n, \delta) \\
& =\int_{0}^{1} W(\pi, \delta) \mathrm{d} U_{1: n}(\pi)-\int_{0}^{1} W(\pi, \delta) \mathrm{d} U_{2: n}(\pi) \\
& =\int_{0}^{1} W(\pi, \delta) \mathrm{d}\left(U_{1: n}(\pi)-U_{2: n}(\pi)\right)
\end{aligned}
$$

We know $U_{1: n}(\pi)=\pi^{n}$ and $U_{2: n}(\pi)=n \pi^{n-1}-(n-1) \pi^{n}$.

$$
\begin{aligned}
U_{1: n}(\pi)-U_{2: n}(\pi) & =n\left(\pi^{n}-\pi^{n-1}\right) \\
\Rightarrow \lim _{n \rightarrow \infty} U_{1: n}(\pi)-U_{2: n}(\pi) & =0
\end{aligned}
$$

As $W(\pi, \delta)$ is bounded and monotone in $\pi$, and $\left(U_{1: n}(\pi)-U_{2: n}(\pi)\right)$ converges to zero then $R_{w}(n, \delta)$ also converges to zero

$$
\lim _{n \rightarrow \infty} R_{w}(n, \delta)=\lim _{n \rightarrow \infty} \int_{0}^{1} W(\pi, \delta) \mathrm{d}\left(n\left(\pi^{n}-\pi^{n-1}\right)\right)=0
$$


As, information eventually becomes valuable, the objective function of the auctioneer approaches total surplus as $n$ goes to infinity.

\section{Proof of Proposition 6:}

$F_{\delta}$ is more stochastically increasing in $\pi$ than $F_{\delta^{\prime}}$ then the Spence-Mirlees type condition (4) holds (Jewitt(1997), Section 5.4). Following an argument presented in LiCalzi (2005):

$$
\begin{aligned}
\frac{\partial}{\partial \delta} W(\pi, \delta) & =\frac{\partial}{\partial \delta} \int_{\mathcal{V}}\left(1-F_{\delta}(v \mid \pi)\right) d v \\
& =-\int_{\mathcal{V}} \frac{\partial}{\partial \delta} F_{\delta}(v \mid \pi) d v \\
& =-\int_{\mathcal{V}} \frac{\partial F_{\delta}(v \mid \pi) / \partial \delta}{\partial F_{\delta}(v \mid \pi) / \partial v} d F_{\delta}(v \mid \pi)
\end{aligned}
$$

As $\frac{\partial F_{\delta}(v \mid \pi) / \partial \delta}{\partial F_{\delta}(v \mid \pi) / \partial v}$ is decreasing in $\pi, \partial W(\pi, \delta) / \partial \delta$ is increasing in $\pi$, i.e. $W(\pi, \delta)$ is supermodular.

\section{Proof of Proposition 7:}

Part (i): We proceed by constructing an example of two information structures ordered in the sense of Blackwell (1951) which are not ordered in the sense of SCprecision.

Let $V$ be uniformly distributed on $[0,1]$. Let $X$ be equal to 0 if $v \in[0,1 / 2)$ and equal to 1 if $v \in[1 / 2,1]$. Similarly, let $Y$ distributed as follows

$$
Y=\left\{\begin{array}{lll}
0 & \text { if } & v \in[0,1 / 4) \\
1 & \text { if } & v \in[1 / 4,1 / 2) \\
2 & \text { if } & v \in[1 / 2,3 / 4) \\
3 & \text { if } & v \in[3 / 4,1]
\end{array}\right.
$$

Clearly $Y$ is sufficient for $X$ so that the information structure for $(V, Y)$, denoted $\mathbf{F}_{\delta}$, is more informative than that for $(V, X)$, denoted $\mathbf{F}_{\delta^{\prime}}$, in the sense of Blackwell. 
Nevertheless, $E[v \mid X=0]=1 / 4$ and $E[v \mid X=1]=3 / 4$, while

$$
E[v \mid Y]=\left\{\begin{array}{lll}
1 / 8 & \text { if } & Y=0 \\
3 / 8 & \text { if } & Y=1 \\
5 / 8 & \text { if } & Y=2 \\
7 / 8 & \text { if } & Y=3
\end{array}\right.
$$

So that if $X$ is the signal from $\mathbf{F}_{\delta^{\prime}}$ and $Y$ with $\mathbf{F}_{\delta}$ then $\psi(\pi) \equiv W(\pi, \delta)-W\left(\pi, \delta^{\prime}\right)$ equals

$$
\psi(\pi)=\left\{\begin{array}{lll}
-1 / 8 & \text { if } & v \in[0,1 / 4) \\
1 / 8 & \text { if } & v \in[1 / 4,1 / 2) \\
-1 / 8 & \text { if } & v \in[1 / 2,3 / 4) \\
1 / 8 & \text { if } & v \in[3 / 4,1]
\end{array}\right.
$$

and $\mathbf{F}_{\delta}$ and $\mathbf{F}_{\delta^{\prime}}$ are not SC-ordered.

Part (ii): We use the following version of the MIO-ND condition in Athey and Levin (2001):

Definition 5 (MIO-ND) The information structure $F_{\delta}$ is more informative than $F_{\delta^{\prime}}$ according to MIO-ND if for all $v \in \mathcal{V}$ and $\pi \in[0,1], F_{\delta^{\prime}}\left(v \mid X \leq F_{\delta^{\prime}}^{-1}(\pi)\right) \leq F_{\delta}(v \mid X \leq$ $\left.F_{\delta}^{-1}(\pi)\right)$

Let $\delta>\delta^{\prime}$. Using the properties of Riemann-Stieltjes integrals we can express $W(\pi, \delta)-W\left(\pi, \delta^{\prime}\right)$ as

$$
W(\pi, \delta)-W\left(\pi, \delta^{\prime}\right)=\int_{\mathcal{V}} v \mathrm{~d} F_{\delta}\left(v \mid F_{\delta}^{-1}(\pi)\right)-\int_{\mathcal{V}} v \mathrm{~d} F_{\delta^{\prime}}\left(v \mid F_{\delta^{\prime}}^{-1}(\pi)\right)
$$

Integrating by parts

$$
W(\pi, \delta)-W\left(\pi, \delta^{\prime}\right)=-\int_{\mathcal{V}}\left(F_{\delta}\left(v \mid F_{\delta}^{-1}(\pi)\right)-F_{\delta^{\prime}}\left(v \mid F_{\delta^{\prime}}^{-1}(\pi)\right)\right) \mathrm{d} v
$$

Then

$$
\int_{0}^{\pi}\left(W(p, \delta)-W\left(p, \delta^{\prime}\right)\right) \mathrm{d} p=-\int_{0}^{\pi}\left(\int_{\mathcal{V}}\left(F_{\delta}\left(v \mid F_{\delta}^{-1}(p)\right)-F_{\delta^{\prime}}\left(v \mid F_{\delta^{\prime}}^{-1}(p)\right)\right) \mathrm{d} v\right) \mathrm{d} p
$$

Interchanging the integration limits

$$
\int_{0}^{\pi}\left(W(p, \delta)-W\left(p, \delta^{\prime}\right)\right) \mathrm{d} p=-\int_{\mathcal{V}} \int_{0}^{\pi}\left(\left(F_{\delta}\left(v \mid F_{\delta}^{-1}(p)\right)-F_{\delta^{\prime}}\left(v \mid F_{\delta^{\prime}}^{-1}(p)\right)\right) \mathrm{d} p\right) \mathrm{d} v
$$


If $\delta$ is more informative according to MIO-ND:

$$
\int_{0}^{\pi}\left(F_{\delta}\left(v \mid F_{\delta}^{-1}(p)\right)-F_{\delta^{\prime}}\left(v \mid F_{\delta^{\prime}}^{-1}(p)\right)\right) \mathrm{d} p \geq 0, \quad \forall \pi \in[0,1]
$$

Hence, we get

$$
\int_{0}^{\pi}\left(W(p, \delta)-W\left(p, \delta^{\prime}\right)\right) \mathrm{d} p \leq 0, \quad \forall \pi \in[0,1]
$$

\section{A.3 Additional Material}

\section{A.3.1 The two examples of dispersion}

Example 1(normal distribution): Consider $\delta>\delta^{\prime}$ and let $\Phi(x)$ be the cumulative distribution of a standard normal distribution, $\phi(x)$ the distribution function:

$$
\begin{aligned}
E[v \mid x] & =\mu+(x-\mu) \frac{\sigma_{v}^{2}}{\sigma_{v}^{2}+\sigma_{\delta}^{2}} \\
F_{\delta}(x) & =\pi=\Phi\left(\frac{x-\mu}{\sqrt{\sigma_{v}^{2}+\sigma_{\delta}^{2}}}\right) \\
\Leftrightarrow F_{\delta}^{-1}(\pi) & =\mu+\Phi^{-1}(\pi)\left(\sqrt{\sigma_{v}^{2}+\sigma_{\delta}^{2}}\right)
\end{aligned}
$$

So that

$$
\begin{aligned}
W(\pi, \delta) & =E\left[v \mid F_{\delta}^{-1}(\pi)\right] \\
& =\mu+\Phi^{-1}(\pi) \frac{\sigma_{v}^{2}}{\sqrt{\sigma_{v}^{2}+\sigma_{\delta}^{2}}} \\
\Rightarrow W(\pi, \delta)-W\left(\pi, \delta^{\prime}\right) & =E\left[v \mid F_{\delta}^{-1}(\pi)\right]-E\left[v \mid F_{\delta^{\prime}}^{-1}(\pi)\right] \\
& =\Phi^{-1}(\pi)\left(\frac{\sigma_{v}^{2}}{\sqrt{\sigma_{v}^{2}+\sigma_{\delta}^{2}}}-\frac{\sigma_{v}^{2}}{\sqrt{\sigma_{v}^{2}+\sigma_{\delta^{\prime}}^{2}}}\right)
\end{aligned}
$$

As $\sigma_{\delta}^{2}<\sigma_{\delta^{\prime}}^{2}$ then

$$
A\left(\delta, \delta^{\prime}\right):=\left(\frac{\sigma_{v}^{2}}{\sqrt{\sigma_{v}^{2}+\sigma_{\delta}^{2}}}-\frac{\sigma_{v}^{2}}{\sqrt{\sigma_{v}^{2}+\sigma_{\delta^{\prime}}^{2}}}\right)>0
$$


so that

$$
\frac{\partial}{\partial \pi}\left(W(\pi, \delta)-W\left(\pi, \delta^{\prime}\right)\right)=\frac{A\left(\delta, \delta^{\prime}\right)}{\phi(\pi)}>0
$$

\section{Example 2(Banerjee):}

Given the structure of the signal, the bidders' expected valuation is

$$
\begin{aligned}
w(x, \delta) & =E[v \mid x] \\
& =x_{i} \delta+(1-\delta) \mu \\
\Rightarrow w(x, \delta)-w\left(x^{\prime}, \delta\right) & =\delta\left(x-x^{\prime}\right) \\
\Leftrightarrow W(\pi, \delta)-W\left(\pi^{\prime}, \delta\right) & =\delta\left(H^{-1}(\pi)-H^{-1}\left(\pi^{\prime}\right)\right)
\end{aligned}
$$

So that for $\pi>\pi^{\prime}, \partial\left(W(\pi, \delta)-W\left(\pi^{\prime}, \delta\right)\right) / \partial \delta>0$.

\section{A.3.2 Further Discussion of Section 5.4}

In this section we provide a more detailed discussion of the statements made in Section 5.4.

Proposition 1 holds under weaker notions of precision, such as integral-precision. Following the arguments in the proof of Proposition 1,

$$
V_{1}(n, \delta) \geq V_{1}\left(n, \delta^{\prime}\right) \Leftrightarrow \int_{0}^{1} \psi(\pi) d U_{1: n}(\pi) \geq 0
$$

where $\psi(\pi) \equiv\left(W(\pi, \delta)-W\left(\pi, \delta^{\prime}\right)\right)$ and let $\Psi(b)=\int_{0}^{b} \psi(\pi) d \pi$. Greater integral precision implies $\Psi(\pi) \leq 0, \forall \pi \in[0,1]$.

Using $U_{1: n}(\pi)=\pi^{n}$ and integrating by parts, equation (6) can be rewritten as

$$
\int_{0}^{1} \psi(\pi) n \pi^{n-1} \mathrm{~d} \pi=-\int_{0}^{1} \Psi(\pi) n(n-1) \pi^{n-2} \mathrm{~d} \pi \geq 0
$$

where the last inequality holds as $\Psi(\pi) \leq 0, \forall \pi \in(0,1)$.

Then, if information provision is costless, the efficient policy is to fully disclose all information - the first part of Theorem 1 trivially holds. 
Also, note that the proof of part (i) of Proposition 3 only makes use of Proposition 1. As this latter result extends to the case where information structures are ordered by integral-precision, then it will also be true that the value of information is negative with only two bidders.

Regardless of the notion of precision, the distribution of the second-order statistic of $n$ independent uniform random variables converges to the distribution of the firstorder statistic and both converge to point mass on $\pi=1$ and integral-precision ensures that $\psi(1)$ is non-negative. This warranties that the optimal amount of information converges to full information as the number of participants goes to infinity.

Integral precision provides limit results but it does not give us any predictions as to the monotonicity of the optimal release of information. On the other hand, SCprecision does provide a sufficient condition for the optimal release of information to be monotone in the number of bidders. The reason is as follows: by SC-precision, $W(\pi, \delta)$ is single-crossing in $(\pi, \delta)$ (in the sense that for all $\delta>\delta^{\prime}, W\left(\pi^{\prime}, \delta\right)-W\left(\pi^{\prime}, \delta^{\prime}\right) \geq 0$ implies for all $\left.\pi>\pi^{\prime}, W(\pi, \delta)-W\left(\pi, \delta^{\prime}\right) \geq 0\right)$. The family of distributions $\left\{U_{2: n}(p)\right\}_{n}$ is ordered by $n$ in the likelihood-ratio order. As

$$
V_{2}(n, \delta)=\int_{0}^{1} W(\pi, \delta) \mathrm{d} U_{2: n}(\pi)
$$

then, by the results in Athey (2002), $V_{2}(n, \delta)$ is single-crossing in $(n, \delta)$ and the optimal choice of $\delta$ is monotone in $n$. 


\section{References}

[1] Athey, S. (2002) "Monotone Comparative Statics under Uncertainty", Quartely Journal of Economics, Vol. 117, pp. 187-223.

[2] Athey, S. and J. Levin (2001) "The Value of Information in Optimal Decision Problems", mimeo.

[3] Baker, E. (2004) "Increasing Risk and Increasing Informativeness: Equivalence Theorems", mimeo.

[4] Banerje, A. (1992) "A Simple Model of Herd Behavior", Quartely Journal of Economics, Vol. 107, pp. 797-817.

[5] Bickel, P. J. and E. L. Lehmann (1976) "Descriptive statistics for nonparametric models. III. Dispersion," Ann. Statist., 4, pp. 1139-1158.

[6] Blackwell, D. (1951) "Comparison of Experiments", Proceedings of the Second Berkeley Symposium on Mathematical Statistics and Probability, J. Neyman, ed. Univ. of California Press, Berkeley, CA, pp. 93-102.

[7] Bergemann, D. and M. Pesendorfer (2003) "Information structures in optimal auctions", mimeo. Updated version of Cowles Foundation Discussion Paper no. 1323, August 2001.

[8] Bergemann, D. and J. Välimäki (2002) "Information Acquisition and Efficient Mechanism Design" Econometrica, 70, 1007-1033.

[9] Board, S. (2004) "Selling Options", working paper, University of Toronto.

[10] Board, S. (2005) "Revealing Information in Auctions: The Efficiency Effect." mimeo. 
[11] Capéraà, P. and Genest, C. (1990) "Concepts de dépendance et orders stochastiques pour des lois bidimensionnelles", Canadian Journal of Statistics, 18, pp $315-326$.

[12] Compte O. and Jehiel, P. (2004) "Auctions and Information Acquisition: Sealedbid or Dynamic Formats?." mimeo.

[13] Eső, P. And Szentes, B. "Optimal Information Disclosure in Auctions and the Handicap Auction", mimeo CMS-EMS Discussion Paper, 2002 (September 2003 version).

[14] Fang Z. and Joe H. (1992) "Further Developments on some dependence orderings for continuous bivariate distributions" Ann. Inst. Statist. Math. 44(3), pp. 501517.

[15] Ganuza, J-J. (2004) "Ignorance promotes competition. An auction model of endogenous private valuations", Rand Journal of Economics, 35, 583-598.

[16] Ganuza, J-J. and Penalva, J.S. (2004) "Optimal Information Transmission in Private Value Auctions", Barcelona Economics Working Paper no. 158.

[17] Jewitt, I. (1997) "Information and Principal Agent Problems", University of Bristol Discussion Paper No. 97/414.

[18] Karlin, S. and Kovikoff, A. (1963) "Generalized Convex Inequalities", Pacific J. Math., 13, 1251-1279.

[19] Kavajecz, K.A. and Keim, D.B. (2004) "Packaging Liquidity: Blind Auctions and Transaction Cost Efficiencies", Journal of Financial and Quantitative Analysis, forthcoming. 
[20] Lehmann, E. (1988) "Comparing Location Experiments," The Annals of Statistics, 16, 521-533.

[21] Lewis, T.R. and Sappington, D.E.M. (1994) "Supplying Information to Facilitate Price Dicrimination ", International Economic review, 35, 309-327.

[22] LiCalzi, M. (2005) "A Sufficient Condition for All-or-nothing Information Supply in Price Discrimination", Rendiconti per gli Estudi Economici e Quantitative, 163-177.

[23] Matthews, S. (1984) "Information acquisition in discriminatory auctions", Bayesian Models in Economic Theory, ed. by M. Boyer and R.E. Kihlstrom. New York: Elsevier Publishers.

[24] Milgrom, P. (1981) "Good News and Bad News: Representation Theorems and Applications", Bell Journal of Economics, 12, 380-391.

[25] Milgrom, P. and C. Shannon (1994) "Monotone comparative statics", Econometrica, 62, 157-180.

[26] Milgrom, P. and R. Weber (1982) "A theory of auctions and competitive bidding", Econometrica, 50, 1089-1122.

[27] Moscarini, G. and Ottaviani, M. "Price Competition for an Informed Buyer", Journal of Economic Theory, Vol. 101 (2001), pp. 457-493.

[28] Ohlin, J. (1969) "On a Class of Measures of Dispersion with Application to Optimal Reinsurance", ASTIN Bulletin, 5(2), 249-266.

[29] Persico, N. (2000) "Information acquisition in auctions", Econometrica, 68, 135149 . 
[30] Shaked, M. and J.G. Shantikumar (1994) Stochastic Orders and their Applications, Academic Press.

[31] Stegeman, M. (1996) "Participation cost and efficient auctions", Journal of Economic Theory, 71, 228-259.

[32] Tan, G (1992) "Entry and R and D in procurement contracting", Journal of Economic Theory, 58, 41-60. 